\title{
Flexible and Robust Privacy-Preserving Implicit Authentication
}

\author{
Josep Domingo-Ferrer ${ }^{1}$, Qianhong $\mathrm{Wu}^{2}$, and Alberto Blanco-Justicia ${ }^{1}$ \\ 1 Universitat Rovira i Virgili \\ Dept. of Computer Engineering and Mathematics \\ UNESCO Chair in Data Privacy \\ Av. Països Catalans 26, E-43007 Tarragona, Catalonia \\ \{josep.domingo, alberto.blanco\}@urv. cat \\ 2 School of Electronics and Information Engineering \\ Beihang University \\ XueYuan Road No. 37, Haidian District, Beijing, China \\ qianhong. wu@buaa.edu.cn
}

\begin{abstract}
Implicit authentication consists of a server authenticating a user based on the user's usage profile, instead of/in addition to relying on something the user explicitly knows (passwords, private keys, etc.). While implicit authentication makes identity theft by third parties more difficult, it requires the server to learn and store the user's usage profile. Recently, the first privacy-preserving implicit authentication system was presented, in which the server does not learn the user's profile. It uses an ad hoc two-party computation protocol to compare the user's fresh sampled features against an encrypted stored user's profile. The protocol requires storing the usage profile and comparing against it using two different cryptosystems, one of them order-preserving; furthermore, features must be numerical. We present here a simpler protocol based on set intersection that has the advantages of: i) requiring only one cryptosystem; ii) not leaking the relative order of fresh feature samples; iii) being able to deal with any type of features (numerical or non-numerical).
\end{abstract}

Keywords: Privacy-preserving implicit authentication, privacy-preserving set intersection, implicit authentication, active authentication, transparent authentication, risk mitigation, data brokers.

\section{Introduction}

The recent report 10 by the U.S. Federal Trade Commission calls for transparency and accountability of data brokers. On the one hand, the report describes the pervasive data collection carried out by data brokers as clearly privacy-invasive. On the other hand, it presents risk mitigation services offered by data brokers as the good side of data collection, to the extent that such services protect consumers against identity theft. Indeed, storing information on how a consumer usually interacts with a service (time of the day, usual places, usual sequence of keystrokes, etc.) allows using this information to implicitly 
authenticate a user: implicit authentication [12] (a.k.a. transparent authentication [5] or active authentication [1]) is the process of comparing the user's current usage profile with the stored profile. If both profiles disagree, maybe someone is impersonating the user, e.g. after some identity theft (password theft, etc.).

The above risk mitigation argument is part a long-standing simplistic tendency in digital services (and elsewhere) to justify privacy invasion in the name of legitimate interests, as if the latter were incompatible with privacy (another old example is intellectual property protection, which was portrayed as being incompatible with the anonymity of digital content consumers until anonymous fingerprinting was proposed [16/8/4]). In fact, implicit authentication turns out to be a weak excuse to justify the storage and/or access by servers to the usage profiles of users. In [17] it was shown how to make implicit authentication compatible with the privacy of users. The idea is that the server only needs an encrypted version of the user's usage profile.

\subsection{Contribution and plan of this paper}

The protocol in [17] needs the server to store the users' accumulated usage profiles encrypted under two different cryptosystems, one that is homomorphic and one that is order-preserving. We present here a protocol for privacy-preserving implicit authentication based on set intersection, which has the advantage that the server only needs to store the users' accumulated usage profiles encrypted under one (homomorphic) cryptosystem. This allows saving storage at the carrier and also computation during the protocol. Also, unlike [17, our protocol does not leak the relative order of fresh feature samples collected by the user's device for comparison with the encrypted profile. Finally, our protocol can deal with any type of features (numerical or non-numerical), while the protocol [17. is restricted to numerical features.

The rest of this paper is organized as follows. Section 2 gives background on implicit authentication and on the privacy-preserving implicit authentication protocol of [17. Section 3 discusses how to compute the dissimilarity between two sets depending on the type of their elements. Section 4 presents a robust privacy-preserving set intersection protocol that can effectively be used for implicit authentication. The privacy, security and complexity of the new protocol are analyzed in Section [5. Experimental results are reported in Section 6. Finally, conclusions and future research directions are summarized in Section 7. The Appendix gives background on privacy-preserving set intersection, recalls the Paillier cryptosystem and justifies the correctness of the least obvious steps of our protocol.

\section{Background}

We first specify the usual setting of implicit authentication and we then move to privacy-preserving implicit authentication. 


\subsection{Implicit authentication}

The usual scenario of implicit authentication is one in which the user carries a mobile networked device (called just user's device in what follows) such as a cell phone, tablet, notebook, etc. The user wishes to authenticate to a server in order to use some application. The user may (or not) use a primary password authentication mechanism. To strengthen such a primary authentication or even to replace it, the user resorts to implicit authentication [12. In this type of authentication, the history of a user's actions on the user's device is used to construct a profile for the user that consists of a set of features. In [12] empirical evidence was given that the features collected from the user's device history are effective to distinguish users and therefore can be used to implicitly authenticate them (instead or in addition to explicit authentication based on the user's providing a password).

The types of features collected on the user's actions fall into three categories: (i) device data, like GPS location data, WiFi/Bluetooth connections and other sensor data; (ii) carrier data, such as information on cell towers seen by the device, or Internet access points; and (iii) cloud data, such as calendar entries. It is not safe to store the accumulated profile of the user in the user's device, because an intruder might compromise the device and alter the stored profile in order to impersonate the legitimate user. Hence, for security, the profile must be stored by some external entity. However, the user's profile includes potentially sensitive information and storing it outside the user's device violates privacy.

Implicit authentication systems try to mitigate the above privacy problem by using a third party, the carrier (i.e. the network service provider) to store the user's profiles. Thus, the typical architecture consists of the user's device, the carrier and the application servers. The latter want to authenticate the user and they collaborate with the carrier and the user's device to do so. The user's device engages in a secure two-party computation protocol with the carrier in order to compare the fresh usage features collected by the user's device with the user's profile stored at the carrier. The computation yields a score that is compared (by the carrier or by the application server) against a threshold, in order to decide whether the user is accepted or rejected. In any case, the application server trusts the score computed by the carrier.

\subsection{Privacy-preserving implicit authentication}

In the privacy-preserving implicit authentication system proposed in [17, the user's device encrypts the user's usage profile at set-up time, and forwards it to the carrier, who stores it for later comparison. There is no security problem because during normal operation the user's device does not store the user's profile (it just collects the fresh usage features). There is no privacy problem either, because the carrier does not see the user's profile in the clear.

The core of proposal 17 is the algorithm for computing the dissimilarity score between two inputs: the fresh sample provided by the user's device and the profile stored at the carrier. All the computation takes place at the carrier 
and both inputs are encrypted: indeed, the carrier stores the encrypted profile and the user's device sends the encrypted fresh sample to the carrier. Note that the keys to both encryptions are only known to the user's device (it is the device who encrypted everything).

The carrier computes a dissimilarity score at the feature level, while provably guaranteeing that: i) no information about the profile stored at the carrier is revealed to the device other than the average absolute deviation of the stored feature values; ii) no information about the fresh feature value provided by the device is revealed to the carrier other than how it is ordered with respect to the stored profile feature values.

The score computation protocol in [17 uses two different encryption schemes: a homomorphic encryption scheme $H E$ (for example, Paillier's [15]) and an order-preserving symmetric encryption scheme OPSE (for example, 4]). For each feature in the accumulated user's profile, two encrypted versions are created, one under $H E$ and the other under OPSE. Similarly, for each feature in the fresh sample it collects, the user's device computes two encrypted versions, under $H E$ and $O P S E$, respectively, and sends them to the carrier. The following process is repeated for each feature:

1. Using the $H E$ ciphertexts the carrier performs some computations (additions and scalar multiplications) relating the encrypted fresh sampled feature value and the set of encrypted feature values in the stored encrypted user's profile.

2. The output of the previous computations is returned to the user's device, which decrypts it, re-encrypts it under $O P S E$ and returns the re-encrypted value to the carrier.

3. Using the order-preserving properties, the carrier can finally compute a dissimilarity score evaluating how different is the fresh sampled feature from those stored in the encrypted user's profile. This score can be roughly described as the number of feature values in the stored encrypted profile that are less dissimilar from the median of the stored values than the fresh sampled value.

The authors of [17] point out that, in case of a malicious user's device (e.g. as a result of it being compromised), one cannot trust the device to provide the correct $H E$-encrypted version of the fresh sampled feature. Nor can it be assumed that the device returns correct $O P S E$-encryptions in Step 2 above. In 17, a variant of the privacy-preserving implicit authentication protocol is presented in which the device proves the correctness of $H E$-encrypted fresh sampled features and does not need to provide $O P S E$-encrypted values. This version is secure against malicious devices, but its complexity is substantially higher.

Other shortcomings of [17:

- It is restricted to numerical features, due to the kind of computations that need to be performed on them. However, among the example features listed in Section 2.1, there are some features that are not numerical, like the list of cell towers or Internet access points seen by the user's device. 
- It discloses the following information to the user's device: i) how the fresh sample is ordered with respect to the stored profile feature values; ii) the average absolute deviation of the stored feature values.

We present a privacy-preserving implicit authentication protocol based on set intersection that deals with the above shortcomings.

\section{Dissimilarity between sets depending on the data type}

Based on [2], we recall here how the dissimilarity between two data sets $X$ and $Y$ can be evaluated using set intersection. If we let $X$ be the user's profile and $Y$ be the fresh sample collected by the user's device, our privacy-preserving implicit authentication setting presents the additional complication (not present in [2]) that $X$ is only available in encrypted form (the carrier stores only the encrypted user's profile). Anyway, we describe here the case of two plaintext sets $X$ and $Y$ and we will deal with encrypted sets in the following sections.

\subsection{Case A: independent nominal feature values}

Assume $X$ and $Y$ consist of qualitative values, which are independent and binary, that is, the relationship between two values is equality or nothing. Take as an example the names of the network or phone providers seen by the user's device, the operating system run by the device and/or the programs installed in the device. In this case, the dissimilarity between $X$ and $Y$ can be evaluated as the multiplicative inverse of the size of the intersection of $X$ and $Y$, that is $1 /|X \cap Y|$, when the intersection is not empty. If it is empty, we say that the dissimilarity is $\infty$.

Clearly, the more the coincidences between $X$ and $Y$, the more similar is the profile stored at the carrier to the fresh sample collected by the device.

\subsection{Case B: correlated categorical feature values}

As in the previous case, we assume the feature values are expressed as qualitative features. However, these may not be independent. For example, if the feature values are the IDs of cell towers or Internet access points seen by the device, nearby cell towers/access points are more similar to each other than distant cell towers/access points.

In this case, the dissimilarity between $X$ and $Y$ cannot be computed as the size of their intersection.

Assume we have an integer correlation function $l: E \times E \mapsto \mathbb{Z}_{+}$that measures the similarity between the values in the sets of features held by the device and the carrier, where $E$ is the domain where the sets of features of both players take values. For nominal features, semantic similarity measures can be used for this purpose [18; for numerical features that take values over bounded and discrete domains, standard arithmetic functions can be used. Assume further that both the device and the carrier know this function $s$ from the very beginning. 
Here the dissimilarity between the set $X$ and the set $Y$ can be computed as

$$
1 /\left(\sum_{x \in X} \sum_{y \in Y} l(x, y)\right)
$$

when the denominator is nonzero. If it is zero, we say that the distance is $\infty$.

\subsection{Case C: numerical feature values}

In this case, we want to compute the dissimilarity between two sets of numerical values based on set intersection. Numerical features in implicit authentication may include GPS location data, other sensor data, etc. Assume $U=\left\{u_{1}, \cdots, u_{t}\right\}$ and $V=\left\{v_{1}, \cdots, v_{t}\right\}$. A way to measure the dissimilarity between $X$ and $Y$ is to compute $\sum_{i=1}^{t}\left|u_{i}-v_{i}\right|$.

\section{Robust privacy-preserving set intersection for implicit authentication}

It will be shown further below that computing dissimilarities in the above three cases $\mathrm{A}, \mathrm{B}$ and $\mathrm{C}$ can be reduced to computing the cardinality of set intersections. Furthermore, this can be done without the carrier revealing $X$ and without the user's device revealing $Y$, as required in the implicit authentication setting. The idea is that, if the dissimilarity stays below a certain threshold, the user is authenticated; otherwise, authentication is refused.

In Appendix $\mathrm{A}$, we give some background on privacy-preserving set intersection protocols in the literature. Unfortunately, all of them assume an honest-butcurious situation, but we need a privacy-preserving set intersection protocol that works even if the adversary is a malicious one: notice that the user's device may be corrupted, that is, in control of some adversary. Hence we proceed to specifying a set intersection protocol that remains robust in the malicious scenario and we apply it to achieving privacy-preserving implicit authentication in Case A. We then extend it to Cases B and C. We make use of Paillier's cryptosystem [15], which is recalled in Appendix B.

\subsection{Implicit authentication in Case A}

Set-up Let the plaintext user's profile be $\left(a_{1}, \cdots, a_{s}\right)$. In this phase, the user's device transfers the encrypted user's profile to the carrier. To do so, the user's device does:

1. Generate the Paillier cryptosystem with public key $p k=(n, g)$ and secret key $s k$.

2. Compute the polynomial $p(x)=\prod_{i=1}^{s}\left(x-a_{i}\right)=p_{0}+p_{1} x+p_{2} x^{2}+\cdots+p_{s} x^{s}$.

3. Compute $\operatorname{Enc}\left(p_{0}\right), \cdots \operatorname{Enc}\left(p_{s}\right)$ where $\operatorname{Enc}\left(p_{i}\right)=g^{p_{i}} r_{i}^{n} \bmod n^{2}$. 
4. Randomly choose $R^{\prime} \in Z_{n^{2}}$. Find $r_{0}^{\prime}, \cdots, r_{s}^{\prime} \in Z_{n^{2}}$ such that

$$
R^{\prime}=r_{0}^{\prime} \cdot r_{1}^{\prime a_{j}} \cdot r_{2}^{\prime a_{j}^{2}} \cdots r_{s}^{a_{j}^{s}} \bmod n^{2}, \quad j=1, \cdots, s
$$

Note that the system (11) has a trivial solution $r_{0}^{\prime}=R^{\prime}$ and $r_{1}^{\prime}=\cdots=r_{s}^{\prime}=1$, but, since it is underdetermined ( $s+1$ unknowns and $s$ equations), it has many non-trivial solutions too (see correctness analysis in Appendix C).

5. Compute $R_{i}=r_{i}^{\prime} / r_{i} \bmod n^{2}$. Randomly choose integer $d \in Z_{n}$. Send

$$
p k, \operatorname{Enc}\left(p_{0}\right), \cdots \operatorname{Enc}\left(p_{s}\right) ; R_{0}{ }^{d}, \cdots, R_{s}{ }^{d} \bmod n^{2}
$$

to the carrier. Locally delete all data computed during the set-up protocol, but keep $\left(d, R^{\prime}\right)$ secretly.

Implicit authentication protocol As discussed in Section 3.1, in case of independent nominal feature values (Case A), dissimilarity is computed as $1 / \mid X \cap$ $Y \mid$. Hence, to perform implicit authentication the carrier just needs to compute the cardinality of the intersection between the fresh sample collected by the user's device and the user's profile stored at the carrier. The challenge is that the carrier only holds the encrypted user's profile and the user's device does no longer hold the plaintext user's profile either in plaintext or ciphertext.

Let $Y=\left\{b_{1}, \cdots, b_{t}\right\} \subseteq E$ be the fresh sample collected by the user's device. Then the device and the carrier engage in the following protocol:

Step 1 The carrier randomly chooses $\theta$, and sends $p k, \operatorname{Enc}\left(p_{0}\right)^{\theta}, \cdots \operatorname{Enc}\left(p_{s}\right)^{\theta}$; $R_{0}{ }^{d}, \cdots, R_{s}{ }^{d}$ to the user's device.

Step 2 The user's device picks a random integer $r(j) \in \mathbb{Z}_{n^{2}}$ for every $1 \leq j \leq t$. The device computes for $1 \leq j \leq t$

$$
\begin{aligned}
\operatorname{Enc}\left(r(j) \cdot d \cdot \theta \cdot p\left(b_{j}\right)\right) & =\operatorname{Enc}\left(p\left(b_{j}\right)\right)^{d \cdot \theta \cdot r(j)} \\
& =\left(\operatorname{Enc}\left(p_{0}\right) \cdots \operatorname{Enc}\left(p_{s}\right)^{b_{j}^{s}}\right)^{d \cdot \theta \cdot r(j)} \\
& =g^{r(j) \cdot d \cdot \theta p\left(b_{j}\right)} \gamma_{j}^{n \cdot d \cdot \theta} \bmod n^{2}
\end{aligned}
$$

where $\gamma_{j}=\left(r_{0} \cdot r_{1} b_{j} \cdot r_{2} b_{j}^{2} \ldots r_{s}^{b_{j}^{s}}\right)^{r(j)} \bmod n^{2}$. The user's device then computes $\Upsilon_{j}=\left(R_{0} \cdot R_{1}{ }^{b_{j}} \cdot R_{2} b_{j}^{2} \cdots R_{s}{ }^{b_{j}^{s}}\right)^{d r(j)} \bmod n^{2}$. For all $j$, the device randomly orders and sends

$$
\left\{\left(\operatorname{Enc}\left(r(j) \cdot d \cdot \theta \cdot p\left(b_{j}\right)\right), \Upsilon_{j}, R^{\prime r(j) d}\right)\right\}
$$

to the carrier.

Step 3 For $1 \leq j \leq t$, the carrier does:

- Compute $\operatorname{Enc}\left(r(j) \cdot d \cdot \theta \cdot p\left(b_{j}\right)\right) \cdot \Upsilon_{j}^{n \theta}$

- From Expression (11), if $b_{j}=a_{i}$ for some $i \in\{1, \cdots, s\}$, then $p\left(b_{j}\right)=0$ and hence $\operatorname{Enc}\left(r(j) d \cdot \theta \cdot p\left(b_{j}\right)\right) \cdot \Upsilon_{j}^{n \theta}=R^{\prime r(j) d n \theta}$; note that the carrier can recognize $R^{\prime r(j) d n \theta}$ by raising $R^{\prime r(j) d}$ received in Expression (2) to $n \theta$. Otherwise (if $b_{j} \neq a_{i}$ for all $i \in\{1, \cdots, s\}$ ) $\operatorname{Enc}\left(r(j) \cdot d \cdot \theta \cdot p\left(b_{j}\right)\right.$ ) looks random. See correctness analysis in Appendix [C]

If both parties are honest, then the carrier learns $|X \cap Y|$ but obtains no information about the elements in $X$ or $Y$. 


\subsection{Implicit authentication in Case B}

Here, the carrier inputs $X$ and the user's device inputs $Y$, two sets of features, and they want to know how close $X$ and $Y$ are without revealing their own set. In the protocol below, only the carrier learns how close $X$ and $Y$ are.

We assume that the domain of $X$ and $Y$ is the same, and we call it $E$. The closeness or similarity between elements is computed by means of a function $s$. In particular, we consider functions $l: E \times E \rightarrow \mathbb{Z}_{+}$. Observe that Case $\mathrm{A}$ is a particular instance of this Case B in which $l(x, x)=1$ and $l(x, y)=0$ for $x \neq y$.

Let $Y$ be the input of the user's device. For every $z \in E$, the device computes $\ell_{z}=\sum_{y \in Y} l(z, y)$. Observe that $\ell_{z}$ measures the overall similarity of $z$ and $Y$. Let $Y^{\prime}=\left\{z \in E: \ell_{z}>0\right\}$. It is common to consider functions satisfying $l(z, z)>0$ for every $z \in E$, and so in general $Y \subseteq Y^{\prime}$.

An implicit authentication protocol for such a computation can be obtained from the protocol in Case A (Section 4.1), by replacing Steps 2 and 3 there with the following ones:

Step 2' For every $z \in Y^{\prime}$, the user's device picks $\ell_{z}$ random integers $r(1), \cdots$, $r\left(\ell_{z}\right) \in \mathbb{Z}_{n^{2}}$ and for $1 \leq j \leq \ell_{z}$ does

- Compute

$$
\begin{aligned}
\operatorname{Enc}(r(j) \cdot d \cdot \theta \cdot p(z)) & =\operatorname{Enc}(p(z))^{d \cdot \theta \cdot r(j)} \\
& =\left(\operatorname{Enc}\left(p_{0}\right) \cdots \operatorname{Enc}\left(p_{s}\right)^{z^{s}}\right)^{d \cdot \theta \cdot r(j)} \\
& =g^{r(j) \cdot d \cdot \theta p(z)} \gamma_{j}^{n \cdot d \cdot \theta} \bmod n^{2}
\end{aligned}
$$

where $\gamma_{j}=\left(r_{0} \cdot r_{1}{ }^{z} \cdot r_{2} z^{2} \cdots r_{s} z^{s}\right)^{r(j)} \bmod n^{2}$.

- Compute $\Upsilon_{j}=\left(R_{0} \cdot R_{1}{ }^{z} \cdot R_{2}{ }^{z^{2}} \cdots R_{s}{ }^{z^{s}}\right)^{d r(j)} \bmod n^{2}$.

- Let $E_{j}=\left\{\left(\operatorname{Enc}(r(j) \cdot d \cdot \theta \cdot p(z)), \Upsilon_{j}, R^{\prime r(j) d}\right)\right\}$.

Finally, the user's device randomly re-orders the sequence of all computed $E_{j}$ for all $z \in Y^{\prime}$ (a total of $\sum_{z \in Y^{\prime}} \ell_{z}$ elements) and sends the randomly re-ordered sequence of $E_{j}$ 's to the carrier.

Step 3' For every received $E_{j}$, the carrier does

- Compute $\operatorname{Enc}(r(j) d \theta \cdot p(z)) \cdot \Upsilon_{j}^{n \theta}$

- From Expression (10), if $z \in X$, then $p(z)=0$ and hence $\operatorname{Enc}(r(j) d \cdot \theta$. $p(z)) \cdot \Upsilon_{j}^{n \theta}=R^{\prime r(j) d n \theta}$ (see correctness analysis in Appendix Cl); otherwise (if $z \notin X) \operatorname{Enc}(r(j) d \theta \cdot p(z))$ looks random.

Hence, at the end of the protocol, the total number of $E_{j}$ which yield $R^{\prime r(j) d n \theta}$ is

$$
\sum_{x \in X} \ell_{x}=\sum_{x \in X} \sum_{y \in Y} l(x, y)
$$

that is, the sum of similarities between the elements of $X$ and $Y$. This clearly measures how similar $X$ and $Y$ are. At the end of the protocol, the carrier knows $\left|Y^{\prime}\right|$ and the device knows $|X|$. Besides that, neither the carrier nor the device can gain any additional knowledge on the elements of each other's set of preferences. 


\subsection{Implicit authentication in Case C}

Let the plaintext user's profile be a set $U$ of $t$ numerical features, which we denote by $U=\left\{u_{1}, \cdots, u_{t}\right\}$. The device's fresh sample corresponding to those features is $V=\left\{v_{1}, \cdots, v_{t}\right\}$. The carrier wants to learn how close $X$ and $Y$ are, that is, $\sum_{i=1}^{t}\left|u_{i}-v_{i}\right|$.

Define $X=\left\{(i, j): u_{i}>0\right.$ and $\left.1 \leq j \leq u_{i}\right\}$ and $Y=\left\{(i, j): v_{i}>0\right.$ and $1 \leq$ $\left.j \leq v_{i}\right\}$. Now, take the set-up protocol defined in Section 4.1 for Case A and run it by using $X$ as plaintext user profile. Then take the implicit authentication protocol for Case A and run it by using $Y$ as the fresh sample input by the device. In this way, the carrier can compute $|X \cap Y|$. Observe that

$$
|X \cap Y|=\mid\left\{(i, j): u_{i}, v_{i}>0 \text { and } 1 \leq j \leq \min \left\{u_{i}, v_{i}\right\}\right\} \mid=\sum_{1 \leq i \leq t} \min \left\{u_{i}, v_{i}\right\} .
$$

In the set-up protocol for Case A, the carrier learns $|X|$ and during the implicit authentication protocol for Case A, the carrier learns $|Y|$. Hence, the carrier can compute

$$
\begin{gathered}
|X|+|Y|-2|X \cap Y|=\sum_{i=1}^{t}\left(\max \left\{u_{i}, v_{i}\right\}+\min \left\{u_{i}, v_{i}\right\}\right)-2 \sum_{i=1}^{t} \min \left\{u_{i}, v_{i}\right\} \\
=\sum_{i=1}^{t}\left(\max \left\{u_{i}, v_{i}\right\}-\min \left\{u_{i}, v_{i}\right\}\right)=\sum_{i=1}^{t}\left|u_{i}-v_{i}\right|
\end{gathered}
$$

\section{$5 \quad$ Privacy, security and complexity}

Unless otherwise stated, the assessment in this section will focus on the protocols of Case A (Section 4.1), the protocols of Cases B and C being extensions of Case A.

\subsection{Privacy and security}

We define privacy in the following two senses:

- After the set-up is concluded, the user's device does not keep any information about the user's profile sent to the carrier. Hence, compromise of the user's device does not result in compromise of the user's profile.

- The carrier learns nothing about the plaintext user's profile, except its size. This allows the user to preserve the privacy of her profile towards the carrier.

Lemma 1. After set-up, the user's device does not keep any information on the user's profile sent to the carrier.

Proof. The user's device only keeps $\left(d, R^{\prime}\right)$ at the end of the set-up protocol. Both $d$ and $R^{\prime}$ are random and hence unrelated to the user's profile. 
Lemma 2. The carrier or any eavesdropper learn nothing about the plaintext user's profile, except its size.

Proof. After set-up, the carrier receives $p k, \operatorname{Enc}\left(p_{0}\right), \cdots \operatorname{Enc}\left(p_{s}\right) ; R_{0}{ }^{d}, \cdots$, $R_{s}{ }^{d} \bmod n^{2}$. Since $d$ is random and unknown to the carrier, $R_{0}{ }^{d}, \cdots, R_{s}{ }^{d}$ $\bmod n^{2}$ look random to the carrier and will give him no more information about the plaintext user's profile than the Paillier ciphertexts $\operatorname{Enc}\left(p_{0}\right), \cdots \operatorname{Enc}\left(p_{s}\right)$. That is, the carrier learns nothing about the user's plaintext profile $X=\left\{a_{1}\right.$, $\left.\cdots, a_{s}\right\}$ except its size $s$. The same holds true for an eavesdropper listening to the communication between the user's device and the carrier during set-up.

At Step 2 of implicit authentication, the carrier only gets the fresh sample $Y$ encrypted under Paillier and randomly re-ordered. Hence, the carrier learns no information on $Y$, except its size $t$. At Step 3, the carrier learns $|X \cap Y|$, but not knowing $Y$, the size $|X \cap Y|$ of the intersection leaks to him no information on $X$.

If we define security of implicit authentication as the inability of a dishonest user's device to disrupt the authentication outcome, we can state the following result.

Lemma 3. A dishonest user's device has no better strategy to alter the outcome of implicit authentication than trying to randomly guess the user's profile.

Proof. At the end of the set-up protocol, the (still uncompromised) user's keeps no information about the user's profile (Lemma 11). Hence, if the user's device is later compromised and/or behaves dishonestly, it still has no clue on the real user's profile against which its fresh sample is going to be authenticated. Hence, either the user's device provides an honest fresh sample and implicit authentication will be correctly performed, or the user's device provides a random fresh sample with the hope that it matches the user's profile.

\subsection{Complexity}

Case A During the set-up protocol, the user's device needs to compute:

$-s+1$ Paillier encryptions for the polynomial coefficients;

- values $r_{0}^{\prime}, \cdots, r_{s}^{\prime}$; as explained in Appendix C, this can be done by randomly choosing $r_{0}^{\prime}$, then solving an $s \times s$ generalized Vandermonde system (doable in $O\left(s^{2}\right)$ time using [7]) and finally computing $s$ modular powers to find the $r_{1}^{\prime}, \cdots, r_{s}^{\prime}$

- $s+1$ modular powers (raising the $R_{i}$ values to $d$ ).

During the implicit authentication protocol, the user's device needs to compute (Step 2):

- $t$ Paillier encryptions;

- $t s$ modular powers (to compute the $\Upsilon_{j}$ values);

- $t$ modular powers (to raise $R^{\prime}$ to $r(j) d$ ). 
Also during the implicit authentication protocol, the carrier needs to compute:

- At Step 1, s+1 modular powers (to raise the encrypted polynomial coefficients to $\theta$ );

- At Step 3, $t$ Paillier encryptions;

- At Step 3, $t$ modular powers (to raise the $\Upsilon_{j}$ values to $n \theta$ ).

Case B The set-up protocol does not change w.r.t. Case A. In the implicit authentication protocol, the highest complexity occurs when $Y^{\prime}=E$ and the similarity function $l$ always takes the maximum value in its range, say $L$. In this case,

$$
\sum_{z \in Y^{\prime}} \ell_{z}=\sum_{z \in Y^{\prime}} \sum_{y \in Y} l(z, y)=|E| s L .
$$

Hence, in the worst case the user's device needs to compute (Step 2'):

- $|E| s L$ Paillier encryptions;

- $|E| s L$ modular powers (to compute the $\Upsilon_{j}$ values);

- $|E| s L$ modular powers (to raise $R^{\prime}$ to $r(j) d$ ).

Also during the implicit authentication protocol, the carrier needs to compute:

- At Step 1, s+1 modular powers (to raise the encrypted polynomial coefficients to $\theta$ );

- At Step 3', $|E| s L$ Paillier encryptions;

- At Step 3', $|E| s L$ modular powers (to raise the $\Upsilon_{j}$ values to $n \theta$ ).

Note that the above complexity can be reduced by reducing the range of the similarity function $l(\cdot, \cdot)$.

Case $\mathbf{C}$ Case $\mathrm{C}$ is analogous to Case A but the sets $X$ and $Y$ whose intersection is computed no longer have $s$ and $t$ elements, respectively. According to Section 4.3, the maximum value for $|X|$ occurs when all $u_{i}$ take the maximum value of their range, say, $M$, in which case $X$ contains $t M$ pairs $(i, j)$. By a similar argument, $Y$ also contains at most $t M$ pairs.

Hence, the worst-case complexity for Case $\mathrm{C}$ is obtained by performing the corresponding changes in the assessment of Case A. Specifically, during the setup protocol, the user's device needs to compute:

$-t M+1$ Paillier encryptions for the polynomial coefficients;

- Solve a Vandermonde system $t M \times t M$ (doable in $O\left((t M)^{2}\right)$ time) and then compute $t M$ modular powers to find the $r_{i}^{\prime}$ values;

- Compute $t M+1$ modular powers (raising the $R_{i}$ values to $d$ ).

During the implicit authentication protocol, the user's device needs to compute (Step 2): 
- $t M$ Paillier encryptions;

- $t^{2} M^{2}$ modular powers (to compute the $\Upsilon_{j}$ values);

- $t M$ modular powers (to raise $R^{\prime}$ to $\left.r(j) d\right)$.

Also during the implicit authentication protocol, the carrier needs to compute:

- At Step 1, $t M+1$ modular powers (to raise the encrypted polynomial coefficients to $\theta$ );

- At Step 3, $t M$ Paillier encryptions;

- At Step 3, $t M$ modular powers (to raise the $\Upsilon_{j}$ values to $n \theta$ ).

Note that the above complexities can be reduced by reducing the range of the numerical values in sets $U$ and $V$.

\section{Experimental results}

As stated in the previous section, the complexity of our implicit authentication protocol ultimately depends on the sizes of the input sets. In Case A, the sizes of the sets are directly given by the user inputs; in Case B, these sizes are the product of the size of the input sets times the range of the similarity function $\ell$; and in Case $\mathrm{C}$, the sizes are given by the size of the original sets times the range of their values. We ran an experiment to test the execution times of our protocol, based on Case A, to which the other two cases can be reduced.

The experiment was implemented in Sage-6.4.1 and run on a Debian7.7 machine with a 64-bit architecture, an Intel i7 processor and 8GB of physical memory. We instantiated a Paillier cryptosystem with a 1024-bit long $n$, and the features of preference sets were taken from the integers in the range $\left[1 \ldots 2^{128}\right]$. The input sets ranged from size 1 to 50 , and we took feature sets of the same size to execute the set-up and the authentication protocols.

Step 4 of the set-up protocol (Section 4.1), in which a system of equations is solved for $r_{i}^{\prime}$ for $1 \leq i \leq s$, is the most expensive part of the set-up protocol. As a worst-case setting, we used straightforward Gaussian elimination which takes time $O\left(s^{3}\right)$, although, as mentioned above, specific methods like [7] exist for generalized Vandermonde matrices that can run in $O\left(s^{2}\right)$ (such specific methods could be leveraged in case of smartphones with low computational power). On the other hand, Step 2 of the authentication protocol (Section 4.1), computed by the user's device, is easily parallelizable for each feature in the sample set. Since parallelization can be exploited by most of the current smartphones in the market, we also exploited it in our experiment. The results are shown in Table 1 (times are in seconds).

Note that the set-up protocol is run only once (actually, maybe once in a while), so it is not time-critical. However, the authentication protocol is to be run at every authentication attempt by the user. For example, if a user implicitly authenticates herself using the pattern of her 20 most visited websites, authentication with our proposal would take 3.37 seconds, which is perfectly acceptable in practice. 
Table 1. Execution times (in seconds) for different input set sizes

\begin{tabular}{|c|c|c|c|c|c|c|c|c|c|c|c|}
\hline & 1 & 5 & 10 & 15 & 20 & 25 & 30 & 35 & 40 & 45 & 50 \\
\hline Set-up & 0.89 & 0.79 & 1.1 & 1.83 & 4.67 & 11.45 & 24.65 & 47.6 & 84.99 & 144.81 & 228.6 \\
\hline Authentication & 0.08 & 0.47 & 1.05 & 2.0 & 3.37 & 5.4 & 8.27 & 12.13 & 17.3 & 23.39 & 31.2 \\
\hline
\end{tabular}

\section{Conclusions and future research}

To the best of our knowledge, we have presented the second privacy-preserving implicit authentication system in the literature (the first one was [17). The advantages of our proposal with respect to [17] are:

- The carrier only needs to store the user's profile encrypted under one cryptosystem, namely Paillier's.

- Dishonest behavior or compromise at the user's device after the initial setup stage neither compromises the privacy of the user's profile nor affects the security of authentication.

- Our proposal is not restricted to numerical features, but can deal also with all sorts of categorical features.

- In case of numerical or categorical ordinal features, our proposal does not disclose how the fresh sample is ordered with respect to the feature values in the stored user's profile.

For binary or independent nominal features, the complexity of our proposal is quite low (quadratic in the number of values in the user's profile). For correlated categorical feature values, the complexity is higher, but it can be reduced by decreasing the range of the similarity function used. Finally, in the case of numerical values, the complexity is also higher than in the binary/independent nominal case, but it can be reduced by decreasing the range of the numerical feature values.

Future research will include devising ways to further decrease the computational complexity in all cases.

\section{Acknowledgments}

The following funding sources are gratefully acknowledged: Government of Catalonia (ICREA Acadèmia Prize to the first author and grant 2014 SGR 537), Spanish Government (project TIN2011-27076-C03-01 "CO-PRIVACY"), European Commission (projects FP7 "DwB", FP7 "Inter-Trust" and H2020 "CLARUS"), Templeton World Charity Foundation (grant TWCF0095/AB60 "CO-UTILITY"), Google (Faculty Research Award to the first author) and Government of China (Natural Science Foundation of China under projects 61370190 and 61173154). The first author is with the UNESCO Chair in Data Privacy. The views in this paper are the authors' own and do not necessarily reflect the views of UNESCO, the Templeton World Charity Foundation or Google. 


\section{References}

1. Y. Aksari, "Active authentication by mouse movements", in 24th Intl. Symposium on Computer and Information Sciences-ISCIS 2009, IEEE, pp. 571-574, 2009.

2. A. Blanco-Justicia, J. Domingo-Ferrer, O. Farràs and D. Sánchez, "Distance computation between two private preference functions", in IFIP SEC 2014-Intl. Information Security and Privacy Conference, IFIP AICT 428, pp. 460-470, 2014.

3. M. Blanton and E. Aguiar, "Private and oblivious set and multiset operations", in ASIACCS 2012, Springer, pp. 40-41, 2012.

4. A. Boldyreva, N. Chenette and A. O'Neill, "Order-preserving symmetric encryption", in EUROCRYPT 2009, Springer, pp. 224-241, 2009.

5. N. Clarke, S. Karatzouni and S. Furnell, "Flexible and transparent user authentication for mobile devices", in IFIP SEC 2009-Intl. Information Security Conference, IFIP AICT 297, pp. 1-12, 2009.

6. E. De Cristofaro, P. Gasti and G. Tsudik, "Fast and private computation of cardinality of set intersection and union", in CANS 2012, Springer, pp. 218-231, 2012.

7. J. Demmel and P. Koev, "The accurate and efficient solution of a totally positive generalized Vandermonde linear system", SIAM Journal on Matrix Analysis and Applications, 27(1):142-152, 2005.

8. J. Domingo-Ferrer, "Anonymous fingerprinting of electronic information with automatic identification of redistributors", Electronics Letters, 34(13):1303-1304, 1998.

9. J. Freedman, K. Nissim and B. Pinkas, "Efficient private matching and set intersection", in EUROCRYPT 2004, Springer, pp. 1-19, 2004.

10. Federal Trade Commission, Data Brokers: A Call for Transparency and Accountability, May 2014.

11. S. Hohenberger and S. Weis, "Honest-verifier private disjointness testing without random oracles", in PET 2006, Springer, pp. 277-294, 2006.

12. M. Jakobsson, E. Shi, P. Golle and R. Chow, "Implicit authentication for mobile devices", in Proc. of the 4th USENIX Conf. on Hot Topics in Security, 2009.

13. L. Kissner and D. X. Song, "Privacy-preserving set operations", in CRYPTO 2005, Springer, pp. 241-257, 2005.

14. D. Megías and J. Domingo-Ferrer, "Privacy-aware peer-to-peer content distribution using automatically recombined fingerprints", Multimedia Systems, 20(2):105-125, 2014.

15. P. Paillier, "Public-key cryptosystems based on composite degree residuosity classes", in EUROCRYPT 1999, Springer, pp. 223-238, 1999.

16. B. Pfitzmann and M. Waidner, "Anonymous fingerprinting", in EUROCRYPT 1997, Springer, pp. 88-102, 1997.

17. N. A. Safa, R. Safavi-Naini and S. F. Shahandashti, "Privacy-preserving implicit authentication", in IFIP SEC 2014-Intl. Information Security and Privacy Conference, IFIP AICT 428, pp. 471-484, 2014.

18. D. Sánchez, M. Batet, D. Isern and A. Valls, "Ontology-based semantic similarity: A new feature-based approach", Expert Systems with Applications, 39(9):7718-7728, 2012.

19. J. Vaidya and C. Clifton, "Secure set intersection cardinality with application to association rule mining", Journal of Computer Security, 13(4):593-622, 2005.

20. A.C.-C. Yao, "How to generate and exchange secrets", in FOCS 1986, pp. 162-167, 1986. 


\section{A Background on privacy-preserving set intersection}

Secure multiparty computation (MPC) allows a set of parties to compute functions of their inputs in a secure way without requiring a trusted third party. During the execution of the protocol, the parties do not learn anything about each other's input except what is implied by the output itself. There are two main adversarial models: honest-but-curious adversaries and malicious adversaries. In the former model, the parties follow the protocol instructions but they try to obtain information about the inputs of other parties from the messages they receive. In the latter model, the adversary may deviate from the protocol in an arbitrary way.

We will restrict here to a two-party setting in which the input of each party is a set, and the desired output is the cardinality of the intersection of both sets. The intersection of two sets can be obtained by using generic constructions based on Yao's garbled circuit [20. This technique allows computing any arithmetic function, but for most of the functions it is inefficient. Many of the recent works on two-party computation are focused on improving the efficiency of these protocols for particular families of functions.

Freedman, Nissim, and Pinkas 9] presented a more efficient method to compute the set intersection, a private matching scheme, that is secure in the honestbut-curious model. A private matching scheme is a protocol between a client $\mathcal{C}$ and a server $\mathcal{S}$ in which $\mathcal{C}$ 's input is a set $X$ of size $i_{\mathcal{C}}, \mathcal{S}$ 's input is a set $Y$ of size $i_{\mathcal{S}}$, and at the end of the protocol $\mathcal{C}$ learns $X \cap Y$. The scheme uses polynomialbased techniques and homomorphic encryption schemes. Several variations of the private matching scheme were also presented in [9: an extension to the malicious adversary model, an extension of the multi-party case, and schemes to compute the cardinality of the set intersection and other functions. Constructing efficient schemes for set operations is an important topic in MPC and has been studied in many other contributions. Several works such as 36[11/13]19 present new protocols to compute the set intersection cardinality.

\section{B Paillier's cryptosystem}

In this cryptosystem, the public key consists of an integer $n$ (product of two RSA primes), and an integer $g$ of order $n$ modulo $n^{2}$, for example, $g=1+n$. The secret key is $\phi(n)$, where $\phi(\cdot)$ is Euler's totient function.

Encryption of a plaintext integer $m$, with $m<n$ involves selecting a random integer $r<n$ and computing the ciphertext $c$ as

$$
c=\operatorname{Enc}(m)=g^{m} \cdot r^{n} \bmod n^{2}=(1+m n) r^{n} \bmod n^{2} .
$$

Decryption consists of first computing $c_{1}=c^{\phi(n)} \bmod n^{2}=1+m \phi(n) n \bmod n^{2}$ and then $m=\left(c_{1}-1\right) \phi(n)^{-1} \bmod n^{2}$.

The homomorphic properties of Paillier's cryptosystem are as follows: 
- Homomorphic addition of plaintexts. The product of two ciphertexts decrypts as the sum of their corresponding plaintexts:

$$
D\left(E\left(m_{1}, r_{1}\right) \cdot E\left(m_{2}, r_{2}\right) \bmod n^{2}\right)=m_{1}+m_{2} \bmod n .
$$

Also, the product of a ciphertext times $g$ raised to a plaintext decrypts as the sum of the corresponding plaintexts:

$$
D\left(E\left(m_{1}, r_{1}\right) \cdot g^{m_{2}} \bmod n^{2}\right)=m_{1}+m_{2} \bmod n .
$$

- Homomorphic multiplication of plaintexts. An encrypted plaintext raised to the power of another plaintext will decrypt to the product of the two plaintexts:

$$
D\left(E\left(m_{1}, r_{1}\right)^{m_{2}} \bmod n^{2}\right)=D\left(E\left(m_{1}, r_{1}\right)^{m_{2}} \bmod n^{2}\right)=m_{1} m_{2} \bmod n .
$$

More generally, given a constant $k, D\left(E\left(m_{1}, r_{1}\right)^{k} \bmod n^{2}\right)=k m_{1} \bmod n$.

\section{Correctness}

In general, the correctness of our protocol follows from direct algebraic verification using the properties of Paillier's cryptosystem. We go next through the least obvious steps.

\section{C.1 Set-up protocol}

In the set-up protocol, $r_{0}^{\prime}, \cdots, r_{s}^{\prime}$ are found as a solution of the following system

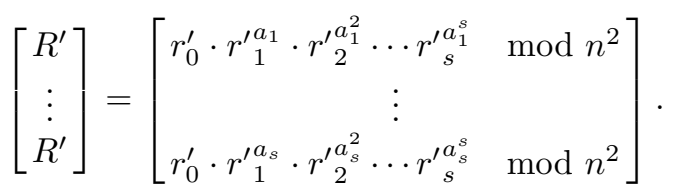

The above system has $s+1$ unknowns and $s$ equations. Therefore it has one degree of freedom. To avoid the trivial solution $r_{0}^{\prime}=R^{\prime}$ and $r_{1}^{\prime}=\cdots=r_{s}^{\prime}=1$, we choose a random $r_{0}^{\prime}$. Then we divide the system by $r_{0}^{\prime}$ and we take logarithms to get

$$
\left[\begin{array}{c}
\log \left(R^{\prime} / r_{0}^{\prime}\right) \\
\log \left(R^{\prime} / r_{0}^{\prime}\right) \\
\vdots \\
\log \left(R^{\prime} / r_{0}^{\prime}\right)
\end{array}\right] \quad \bmod n=\left[\begin{array}{cccc}
a_{1} & a_{1}^{2} & \cdots & a_{1}^{s} \\
\vdots & \vdots & \vdots & \\
a_{s} & a_{s}^{2} & \cdots & a_{s}^{s}
\end{array}\right] \cdot\left[\begin{array}{c}
\log r_{1}^{\prime} \\
\log r_{2}^{\prime} \\
\vdots \\
\log r_{s}^{\prime}
\end{array}\right] \quad \bmod n
$$

The matrix on the right-hand side of the above system is an $s \times s$ generalized Vandermonde matrix (not quite a Vandermonde matrix). Hence, using the techniques in [7] it can be solved in $O\left(s^{2}\right)$ time for $\log r_{1}^{\prime}, \cdots, \log r_{s}^{\prime}$. Then $s$ powers modulo $n^{2}$ need to be computed to turn $\log r_{i}^{\prime}$ into $r_{i}^{\prime}$ for $i=0, \cdots, s$. 


\section{C.2 Implicit authentication protocol}

We specify in more detail the following derivation in Step 2 of the implicit authentication protocol of Section 4.1.

$$
\begin{aligned}
\operatorname{Enc}\left(r(j) \cdot d \cdot \theta \cdot p\left(b_{j}\right)\right) & =\operatorname{Enc}\left(p\left(b_{j}\right)\right)^{d \cdot \theta \cdot r(j)} \bmod n^{2} \\
& =\left(\operatorname{Enc}\left(p_{0}\right) \cdots \operatorname{Enc}\left(p_{s}\right)^{b_{j}^{s}}\right)^{d \cdot \theta \cdot r(j)} \bmod n^{2} \\
& =\left(g^{p_{0}} r_{0}^{n} \cdots\left(g^{p_{s}} r_{s}^{n}\right)^{b_{j}^{s}}\right)^{d \cdot \theta \cdot r(j)} \bmod n^{2} \\
& =\left(g^{p\left(b_{j}\right)}\right)^{d \cdot \theta \cdot r(j)}\left(r_{0} \cdot r_{1}^{b_{j}} \cdots r_{s}^{b_{j}^{s}}\right)^{r(j) \cdot n \cdot d \cdot \theta} \bmod n^{2} \\
& =g^{r(j) \cdot d \cdot \theta p\left(b_{j}\right)} \gamma_{j}^{n \cdot d \cdot \theta} \bmod n^{2} .
\end{aligned}
$$

Regarding Step 3 of the implicit authentication protocol, we detail the case $b_{j}=a_{i}$ for some $i \in\{1, \cdots, s\}$. In this case, $p\left(b_{j}\right)=0$ and hence

$$
\begin{gathered}
\operatorname{Enc}\left(r(j) d \theta \cdot p\left(b_{j}\right)\right) \cdot \Upsilon_{j}^{n \theta} \bmod n^{2}=\operatorname{Enc}(0)^{r(j) d \theta} \cdot \Upsilon_{j}^{n \theta} \bmod n^{2} \\
=\left(r_{0} \cdot r_{1}^{b_{j}} \cdots r_{s}^{b_{j}^{s}}\right)^{n r(j) d \theta} \cdot \Upsilon_{j}^{n \theta} \bmod n^{2} \\
=\left(r_{0} \cdot r_{1}^{b_{j}} \cdots r_{s}^{b_{j}^{s}}\right)^{n r(j) d \theta} \cdot\left(R_{0} \cdot R_{1}^{b_{j}} \cdots R_{s}^{b_{j}^{s}}\right)^{d r(j) n \theta} \bmod n^{2} \\
=\left(r_{0}^{\prime} \cdot r^{\prime a_{i}} \cdots{r^{\prime}}_{s_{i}^{s}}^{a^{s}}\right)^{r(j) d n \theta} \bmod n^{2}=R^{\prime r(j) d n \theta} \bmod n^{2} .
\end{gathered}
$$

If in Step 3, if we have $b_{j} \neq a_{i}$ for all $i \in\{1, \cdots, s\}$, then Derivation (3) does not hold and a random number is obtained instead. On the one side, the powers of $g$ does not disappear from $\operatorname{Enc}\left(r(j) d \theta \cdot p\left(b_{j}\right)\right)$. On the other side, the exponents $b_{j}, \cdots, b_{j}^{s}$ cannot be changed by $a_{i}, \cdots, a_{i}^{s}$ as done in the last step of Derivation (3). Hence, a random number different from $R^{\prime r(j) d n \theta}$ is obtained. 